\title{
Correction to: Measuring the sustainability of Russia's Arctic cities
}

\author{
Robert W. Orttung $\mathbb{1 0}$, Oleg Anisimov, Svetlana Badina, \\ Charlene Burns, Leena Cho, Benjamin DiNapoli, Matthew Jull, \\ Melissa Shaiman, Ksenia Shapovalova, Leah Silinsky, \\ Emily Zhang, Yelena Zhiltcova
}

Published online: 20 December 2021

\section{Correction to: Ambio (2021) 50:2090-2103 \\ https://doi.org/10.1007/s13280-020-01395-9}

In the original publication, the incomplete affiliation information of the authors Svetlana Badina, Ksenia Shapovalova and Yelena Zhiltcova was processed during the typesetting and publication process. The complete information of the authors is provided in this correction.

The original article has been corrected.

Publisher's Note Springer Nature remains neutral with regard to jurisdictional claims in published maps and institutional affiliations.

Robert W. Orttung ( $\square)$

Address: IERES, 1957 E St NW Suite 412, Washington, DC 20052, USA.

e-mail: rorttung@gwu.edu

\section{Oleg Anisimov}

Address: State Hydrological Institute, 23 Second Line V.O., St.Petersburg, Russia 199053.

e-mail: oleg@oa7661.spb.edu

\section{Svetlana Badina}

Address: Plekhanov Russian University of Economics, 36 Stremyanny lane, Moscow, Russia 117997.

Address: Faculty of Geography, Lomonosov Moscow State University, 1 Leninskie gory, GSP-1, Moscow, Russia 119991.

e-mail: bad412@yandex.ru

\section{Charlene Burns}

Address: IERES, 1957 E St NW Suite 412, Washington, DC 20052, USA.

e-mail: burnsc@gwu.edu

\section{Leena Cho}

Address: UVA School of Architecture, 110 Bayly Drive, Charlottesville, VA 22903, USA.

e-mail: 1cho@virginia.edu

\section{Benjamin DiNapoli}

Address: 48 Quincy St, Cambridge, MA 02138, USA.

e-mail: dinapoli@gsd.harvard.edu

\section{Matthew Jull}

Address: UVA School of Architecture, 110 Bayly Drive, Charlottesville, VA 22903, USA.

e-mail: mjull@virginia.edu

\section{Melissa Shaiman}

Address: IERES, 1957 E St NW Suite 412, Washington, DC 20052, USA.

e-mail: melishaiman@gwu.edu

\section{Ksenia Shapovalova}

Address: State Hydrological Institute, 23 Second Line V.O., St.Petersburg, Russia 199053.

e-mail: X-sha@yandex.ru

Leah Silinsky

Address: IERES, 1957 E St NW Suite 412, Washington, DC 20052, USA.

e-mail: leahsilinsky@gwmail.gwu.edu

\section{Emily Zhang}

Address: IERES, 1957 E St NW Suite 412, Washington, DC 20052, USA.

e-mail: ezhang@gwu.edu

\section{Yelena Zhiltcova}

Address: State Hydrological Institute, 23 Second Line V.O., St.Petersburg, Russia 199053.

e-mail: el-spb@mail.ru 